Canadian Oncology

Nursing Journal

Revue canadienne

de soins infirmiers

en oncologie

Volume 28, Issue 4 • Fall 2018

elSSN: 2368-8076 


\title{
Néoplasies myéloprolifératives - Partie I : survol du diagnostic et du traitement des NMP « classiques »
}

\author{
par Sabrina Fowlkes, Cindy Murray, Adrienne Fulford, Tammy De Gelder et Nancy Siddiq
}

\section{RÉSUMÉ}

Les néoplasies myéloprolifératives (NMP) sont des troubles rares, mais potentiellement mortels causés par la production excessive de cellules souches dans la moelle osseuse. Chez les patients atteints de NMP sans mutation du gène BCR-ABL1 (c.-à-d. la thrombocytémie essentielle [TE], la polycythémie vraie [PV] et la myélofibrose $[M F]$, aussi appelées "NMP classiques »), les symptômes peuvent être incapacitants et nuire à la qualité de vie. Malgré tout, il est possible pour les patients souffrant de ces types de NMP de vivre plusieurs années, moyennant un traitement et un suivi à long terme. Les infirmières jouent donc un rôle essentiel pour favoriser la communication avec ces patients, évaluer leurs symptômes et leur enseigner les traitements et les stratégies d'autosoins pouvant les apaiser. Le présent article, premier d'une série de deux, propose aux infirmières et autres professionnels de la santé une revue du diagnostic et du traitement des NMP classiques les plus fréquentes. Le second article (aussi publié dans ce numéro) présentera aux infirmières des conseils pratiques pour traiter la symptomatologie associée aux NMP et ainsi améliorer la santé et le bien-être général des patients.

\section{AU SUJET DES AUTEURES}

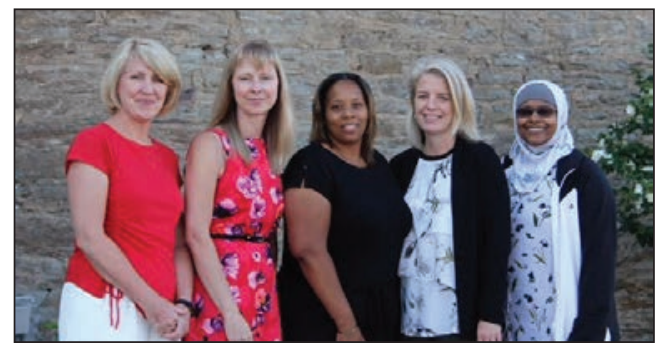

Sur la photo, de gauche à droit : Cindy Murray, Adrienne Fulford, Sabrina Fowlkes, Tammy De Gelder, Nancy Siddiq

Sabrina Fowlkes, inf. aut., B.Sc.inf., clinicienne, leucémie myélö̈de chronique/ néoplasies myéloprolifératives, Hôpital général juif, Montréal, Québec

Cindy Murray, M.Sc.inf., IP (adultes), infirmière praticienne, Hémopathies malignes, Réseau universitaire de santé, Centre de cancérologie Princess Margaret, Toronto, Ontario

Adrienne Fulford, IP-SSP, M.Sc.inf, CSIO(C), infirmière praticienne, Hématooncologie, Centre des sciences de la santé de London, London, Ontario

Tammy De Gelder, M.Sc.inf., IP (adultes), CSIO(C), infirmière praticienne, Centre des sciences de la santé d'Hamilton, Centre hospitalier et de cancérologie Juravinski, Hamilton, Ontario

Nancy Siddiq, inf. aut., B.Sc.inf., CSIO(C), M.Sc.inf./éducation, infirmière clinicienne spécialisée en néoplasies myéloprolifératives, Centre de cancérologie Princess Margaret, Toronto, Ontario

Auteure-ressource : Cindy Murray, M.Sc.inf., IP (adultes), 610 University Ave., Toronto, Ontario M5G 2C4.

Téléphone : 416-946-4501, poste 5919

Courriel : Cindy.Murray@uhn.ca
Mots clés : néoplasies myéloprolifératives, thrombocytémie essentielle, polycythémie vraie, myélofibrose, diagnostic, traitement, pratique infirmière

\section{INTRODUCTION}

L es néoplasies myéloprolifératives (NMP) sont un groupe de maladies rares, mais potentiellement mortelles présentant plusieurs points communs : elles sont causées par une production excessive de cellules souches dans la moelle osseuse. Le dérèglement de la production médullaire de cellules sanguines (hématopoïèse) entraîne un excès ou une insuffisance de cellules sanguines. L'Organisation mondiale de la Santé (OMS) classe les NMP selon la mutation du gène de fusion BCR-ABL1 (voir la figure 1) (Arber et al., 2016). La leucémie myéloïde chronique (LMC) est le trouble le plus couramment associé à la mutation positive du gène BCR-ABL1, dont le traitement a déjà fait l'objet de nombreuses recherches. Le présent article se penchera donc plutôt sur les NMP les plus communes sans mutation du gène BCR-ABL1. Ces dernières sont de gravité variable : certaines formes sont modérées alors que d'autres sont agressives. En outre, les symptômes débilitants et le risque accru d'accidents thrombotiques peuvent sévèrement affecter la qualité de vie. Vu la myriade de ressources accessibles en ligne, la plupart des patients souffrant de NMP connaissent bien leur maladie et les traitements existants. Il importe donc pour les infirmières de posséder une solide compréhension de ces troubles, de même que des interventions thérapeutiques appropriées. Cet article a été rédigé par des infirmières praticiennes canadiennes et des infirmières spécialisées en hémato-oncologie. Il vise à présenter aux infirmières et autres professionnels de la santé une revue des caractéristiques des NMP à « BCR-ABL1 négatif » les plus courantes, des aspects diagnostiques et thérapeutiques, ainsi que du rôle des infirmières dans le traitement. Un second article (aussi publié dans ce numéro) proposera aux infirmières des conseils pratiques pour traiter la symptomatologie associée aux NMP dites « classiques ».

\section{QUELLES SONT LES FORMES LES PLUS COURANTES DE NMP SANS MUTATION DU GÈNE BCR-ABL1?}

Les NMP sans mutation du gène BCR-ABL1 les plus courantes (aussi appelées NMP classiques [voir la figure 1]) sont la thrombocytémie essentielle (TE), la polycythémie vraie (PV) et la myélofibrose (MF). Le tableau 1 résume les principales caractéristiques, de même que les indications cliniques et moléculaires de ces NMP classiques.

La TE représente environ $25 \%$ des NMP classiques (Rollison et al., 2008). Elle est caractérisée par une 


\section{Néoplasies myéloprolifératives (NMP) (Classification de l'OMS)}

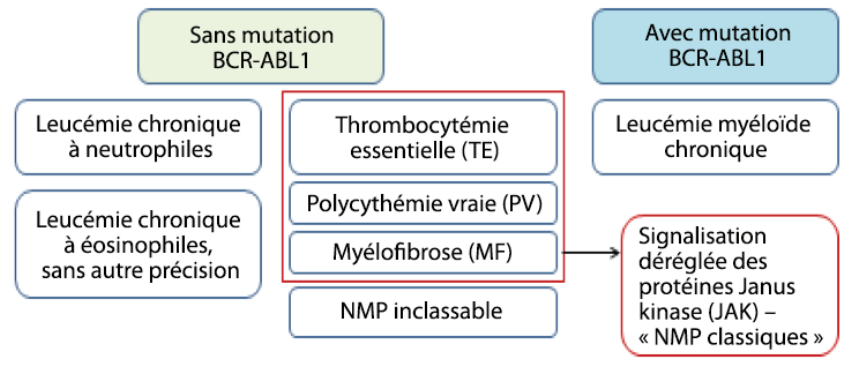

Figure 1 : Aperçu des NMP

thrombocytose (élévation soutenue des plaquettes dans le sang) et une prolifération des mégacaryocytes matures (cellules de la moelle osseuse responsables de la production de plaquettes) (Arber et al., 2016; Tefferi et Barbui, 2015). Dans cette pathologie, la production excessive de plaquettes entraîne parfois des complications thrombotiques et hémorragiques. Parmi les NMP classiques, c'est toutefois la TE qui présente le pronostic le plus favorable, avec une médiane de survie variant entre 18 et 20 ans. Elle tend à toucher davantage les femmes que les hommes (Wolanskyj et al., 2006; Radaelli et al., 2008).

La PV est la NMP la plus fréquente, avec $45 \%$ des cas (Rollison et al., 2008). Caractérisée par une augmentation du volume de globules rouges, elle entraîne une élévation du taux d'hémoglobine et d'hématocrite, ainsi que de la masse des globules rouges (Tonkin et al., 2012). Elle peut également être associée à une leucocytose (augmentation du nombre de globules blancs) et à une thrombocytose (augmentation du nombre de plaquettes), ainsi qu'à une splénomégalie (hypertrophie de la rate) (Arber et al., 2016; Finazzi et Barbui, 2007). La PV accroît également le risque de thrombose. La médiane de survie des personnes atteintes de PV est d'environ 15 à 20 ans et varie selon la présence de certains facteurs pronostiques augmentant le risque de thrombose et de décès, c'est-à-dire l'âge (plus de 60 ans) ainsi que les antécédents de thrombose veineuse et de leucocytose (Tefferi et al., 2013). Environ 10 à $20 \%$ des patients ayant reçu un diagnostic de PV souffriront éventuellement de MF et, dans 2 à $7 \%$ des cas, la maladie évoluera en leucémie myéloïde chronique (LMC) (Passamonti et al., 2011).

La MF est caractérisée par des symptômes débilitants, une splénomégalie et une fibrose progressive de la moelle osseuse par formation de tissu cicatriciel (Abdel-Wahab et Levine, 2009; Tonkin et al., 2012). Cette fibrose entrave la production de globules rouges. La MF peut être primaire (MFP) ou secondaire à une TE (MF post-TE) ou encore à une PV (MF post-PV). Pour les trois formes, les manifestations sont pratiquement identiques et le traitement est généralement le même. La MF évolue fréquemment vers la LMC et, en général, les patients atteints de MF tendent à avoir une espérance de vie plus courte que les patients souffrant de PV ou de TE (Geyer et Mesa, 2014). Toutefois, le pronostic varie selon le niveau de risque du patient (faible, modéré-1, modéré-2 et élevé) pour différents facteurs (voir le tableau 2). Au moment du diagnostic, le score pronostique international (International Prognostic Scoring System IPSS) peut être utilisé pour estimer le pronostic des patients atteints de MF. Une fois le diagnostic posé et pour toute la durée de la maladie, l'évaluation du pronostic peut se faire à l'aide de l'échelle IPSS dynamique (DIPSS) (Cervantes et al., 2009; Passamonti et al., 2010). Les systèmes IPSS et DIPSS établissent

Tableau 1 : Caractéristiques, signes cliniques et déterminants moléculaires

\begin{tabular}{|c|c|c|c|}
\hline & TE & PV & MF \\
\hline $\begin{array}{l}\text { Anomalies/ } \\
\text { mutations } \\
\text { moléculaires }\end{array}$ & $\begin{array}{l}\text { - Mutation JAK2 : 50 à } 60 \% \\
\text { - Mutations MPL : 3 à } 5 \% \\
\text { - Mutation CALR (exon 9) : } 25 \%\end{array}$ & $\begin{array}{l}\text { - Mutation JAK2 : > } 95 \% \\
\text { - Mutations exon } 12 \text { aussi } \\
\text { observées }\end{array}$ & $\begin{array}{l}\text { - Mutation JAK2 : 50\% } \\
\text { - Mutations MPL : 11\% }\end{array}$ \\
\hline $\begin{array}{l}\text { Résultats } \\
\text { cliniques et de } \\
\text { laboratoire }\end{array}$ & $\begin{array}{l}\text { - Généralement asymptomatique } \\
\text { - Prédisposition pour les événements occlusifs } \\
\text { vasculaires (p. ex. thrombose veineuse profonde, } \\
\text { maladie vasculaire périphérique, etc.) } \\
\text { - Hémorragie } \\
\text { - Possibilité d'une splénomégalie discrète } \\
\text { - Symptômes courants : faiblesse, céphalée, } \\
\text { paresthésie des extrémités (neuropathie } \\
\text { périphérique), goutte }\end{array}$ & $\begin{array}{l}\text { - Souvent asymptomatique } \\
\text { - Augmentation des } \\
\text { taux d'hémoglobine et } \\
\text { d'hématocrite } \\
\text { - Thrombose } \\
\text { - Symptômes courants au stade } \\
\text { avancé : prurit, perte de poids, } \\
\text { faiblesse, sueurs nocturnes, } \\
\text { ostéalgie, splénomégalie }\end{array}$ & $\begin{array}{l}\text { - Anémie très fréquente } \\
\text { - Splénomégalie } \\
\text { - Leucoérythroblastose } \\
\text { - Leucocytose ou leucopénie } \\
\text { - Thrombocytose ou } \\
\text { thrombocytopénie } \\
\text { - Symptômes courants : fatigue, } \\
\text { prurit, perte de poids, faiblesse, } \\
\text { sueurs nocturnes, ostéalgie, } \\
\text { splénomégalie }\end{array}$ \\
\hline
\end{tabular}

TE : thrombocytémie essentielle; PV : polycythémie vraie; MF : myélofibrose; JAK2 : Janus kinase 2; MPL : myoloproliferative leukemia (leucémie myéloproliférative); CALR : calréticuline 
leur score à l'aide des mêmes facteurs de risque, mais diffèrent légèrement quant au poids qu'ils accordent à ces facteurs. Une mise à jour récente de l'algorithme (DIPSS-PLUS) permet d'incorporer d'autres facteurs de risque indépendants (Gangat et al., 2011) (voir le tableau 2). Son utilisation n'est toutefois pas encore aussi répandue que celle du score DIPSS. Bien que le taux de survie général des patients atteints de MF à faible risque ( 0 point) et à risque modéré 1 ( 1 ou 2 points) soit prometteur, il diminue beaucoup chez les patients à risque modéré 2 et à risque élevé (voir le tableau 3).

\section{DIAGNOSTIC DES NMP}

Établir le diagnostic des NMP est souvent difficile. En effet, la pathogenèse et les symptômes de la MF, de la PV et de la TE se ressemblent. Le diagnostic est généralement posé par un hématologue qui, habituellement, prescrira des analyses sanguines et moléculaires, ainsi qu'une biopsie de la moelle osseuse.

Le diagnostic différentiel des NMP est important, car il oriente la prise en charge des patients. L'Organisation

Tableau 2 : Systèmes de score pronostique pour la MF dans la pratique clinique actuelle (Cervantes et al., 2009; Passamonti et al., 2010; Gangat et al., 2011)

\begin{tabular}{|c|c|c|c|}
\hline Variable & IPSS & DIPSS & DIPSS-Plus \\
\hline$\hat{A} g e:>65$ ans & $\checkmark$ & $\checkmark$ & $\checkmark$ \\
\hline $\begin{array}{l}\text { Symptômes } \\
\text { constitutionnels }\end{array}$ & $\checkmark$ & $\checkmark$ & $\checkmark$ \\
\hline $\mathrm{Hb}:<100 \mathrm{~g} / \mathrm{L}$ & $\checkmark$ & $\checkmark$ & $\checkmark$ \\
\hline $\begin{array}{l}\text { Leucocytémie: } \\
>25 \times 109 / \mathrm{L}\end{array}$ & $\checkmark$ & $\checkmark$ & $\checkmark$ \\
\hline Blastes dans le sang: $\geq 1 \%$ & $\checkmark$ & $\checkmark$ & $\checkmark$ \\
\hline $\begin{array}{l}\text { Numération plaquettaire : } \\
<100 \times 109 / \mathrm{L}\end{array}$ & & & $\checkmark$ \\
\hline Transfusion de GR & & & $\checkmark$ \\
\hline Caryotype non favorable* & & & $\checkmark$ \\
\hline Score & $\begin{array}{l}1 \text { point } \\
\text { par } \\
\text { élément }\end{array}$ & $\begin{array}{l}1 \text { point par } \\
\text { élément, } \\
\text { mais } \mathrm{Hb}=2\end{array}$ & $\begin{array}{l}1 \text { pt pour } \\
\text { DIPSS mod-1 } \\
2 \text { pts pour } \\
\text { DIPSS mod-2 } \\
3 \text { pts pour } \\
\text { DIPSS élevé } \\
1 \text { point pour } \\
\text { chacun des } \\
\text { trois derniers } \\
\text { éléments }\end{array}$ \\
\hline \multicolumn{4}{|c|}{$\begin{array}{l}{ }^{*} \text { Caryotype non favorable }=8,7 / 7 q \text {, i }(17 q) \text {, inv }(3), 5 / 5 q, 12 p \text {, ou } \\
\text { réarrangements } 11 q 23 \\
\text { IPSS : International Prognostic Scoring System (score } \\
\text { pronostique international); DIPSS : IPSS dynamique; Hb : } \\
\text { hémoglobine; GR : globules rouges. }\end{array}$} \\
\hline
\end{tabular}

mondiale de la Santé (OMS) a défini des critères diagnostiques pour faciliter la différenciation des NMP classiques dans la pratique clinique (Arber et al., 2016). Le tableau 4 présente un schéma simplifié des critères diagnostiques de l'OMS publiés en 2016. La mutation du gène Janus kinase 2 (JAK2), qui produit la tyrosine kinase et participe à la formation des cellules sanguines à partir des cellules souches hématopoiétiques de la moelle osseuse, sert de marqueur moléculaire des NMP. Plus de $95 \%$ des patients souffrant de PV et environ 50 à $60 \%$ des patients atteints de TE et de MF présentent une mutation JAK2 (Nangalia et Green, 2014). Par conséquent, le génotypage du gène JAK2 intervient souvent dans le processus diagnostique des NMP classiques.

\section{TRAITEMENT DES NMP ET OBJECTIFS THÉRAPEUTIQUES}

Le traitement des NMP vise globalement à prévenir les complications thrombotiques et hémorragiques, à contrôler au mieux les symptômes, à bonifier la qualité de vie des patients et à prolonger leur vie. À l'heure actuelle, l'unique approche curative de la MF est la greffe de cellules souches hématopoiétiques. Toutefois, comme la procédure pose un risque considérable de mortalité et de morbidité, elle est généralement réservée aux jeunes patients qui présentent peu de comorbidités et dont la maladie est avancée.

Selon le type de NMP, le traitement intègre parfois des interventions pharmacologiques comme l'acide acétylsalicylique (ASA), l'hydroxyurée, l'interféron alpha, l'anagrélide, le busulfan et le ruxolitinib, ou alors des procédures chirurgicales comme la phlébotomie et la splénectomie (tableau 5). La phlébotomie est pratiquée pour contrôler l'érythrocytose (augmentation de la masse de globules rouges) tout en maintenant le niveau d'hématocrite inférieur à $45 \%$. Les effets secondaires de la phlébotomie sont habituellement minimes, mais certains patients souffrent parfois d'ecchymoses au site de l'intervention et ressentent de la fatigue ou une faiblesse (Tonkin et al., 2012). La splénectomie est l'ablation chirurgicale de la rate. Elle est recommandée uniquement chez les patients souffrant de splénomégalie. Les modalités de traitement non pharmacologiques des NMP n'ont pas fait l'objet de moult publications, à l'exception de la splénectomie et la phlébotomie. La présente section traitera donc principalement des interventions pharmacologiques. Tous les traitements présentés ici sont administrés en clinique externe.

Tableau 3 : Survie en fonction de la classification DIPSS (Passamonti et al., 2010)

\begin{tabular}{|l|l|l|}
\hline & Points & $\begin{array}{l}\text { Médiane de survie } \\
\text { (en mois) }\end{array}$ \\
\hline Faible & 0 & Non encore atteinte \\
\hline Modéré-1 & $1-2$ & 170 \\
\hline Modéré-2 & $3-4$ & 48 \\
\hline Élevé & $5-6$ & 18 \\
\hline
\end{tabular}


Tableau 4 : Critères diagnostiques simplifiés des NMP classiques établis par l'OMS en 2016 (Arber et al., 2016)

\begin{tabular}{|c|c|c|}
\hline TE & PV & MF \\
\hline $\begin{array}{l}\text { Critères majeurs : } \\
\text { 1. Numération plaquettaire élevée } \\
\text { 2. Prolifération de grands mégacaryocytes } \\
\text { matures } \\
\text { 3. Absence des critères suivants : LCM avec } \\
\text { mutation BCR-ABL1, PV, MFP, SMD ou } \\
\text { autres néoplasies myéloïdes } \\
\text { 4. Présence de mutation JAK2, CALR ou } \\
\text { MPL }\end{array}$ & $\begin{array}{l}\text { Critères majeurs : } \\
\text { 1. Hémoglobine ou hématocrite } \\
\text { élevé ou diminution de la masse } \\
\text { de globules rouges* } \\
\text { 2. Moelle osseuse hypercellulaire } \\
\text { avec prolifération touchant les } \\
\text { trois lignées } \\
\text { 3. Présence de mutation 617F ou } \\
\text { exon } 12 \text { de JAK2 }\end{array}$ & $\begin{array}{l}\text { Critères majeurs : } \\
\text { 1. Prolifération de mégacaryocytes atypiques, } \\
\text { accompagnée soit d'une fibrose réticulinique, soit } \\
\text { d'une fibrose collagénique } \\
\text { 2. Absence des critères suivants: TE, PV, LCM avec } \\
\text { mutation BCR-ABL1, SMD ou autres néoplasies } \\
\text { myéloïdes } \\
\text { 3. Présence de mutation JAK2, CALR ou MPL ou, } \\
\text { si ce n'est pas le cas, d'un autre marqueur clonal } \\
\text { ou absence de MF réactionnelle. }\end{array}$ \\
\hline $\begin{array}{l}\text { Critère mineur: } \\
\text { - Présence d'un marqueur clonal ou absence } \\
\text { de signe de thrombocytose réactionnelle }\end{array}$ & $\begin{array}{l}\text { Critère mineur: } \\
\text { - Taux d'érythropoiétine sous la } \\
\text { normale }\end{array}$ & $\begin{array}{l}\text { Critère mineur: } \\
\text { Présence d'au moins un des éléments suivants : } \\
\text { - anémie } \\
\text { - leucocytose } \\
\text { - splénomégalie palpable } \\
\text { - LDH élevée } \\
\text { - leucoérythroblastose }\end{array}$ \\
\hline $\begin{array}{l}\text { Exigences pour le diagnostic: } \\
\text { Les } 4 \text { critères majeurs } \bigcirc \cup 3 \text { critères majeurs } \\
+ \text { le critère mineur }\end{array}$ & $\begin{array}{l}\text { Exigences pour le diagnostic: } \\
\text { Les } 3 \text { critères majeurs } O \cup 2 \text { critères } \\
\text { majeurs + le critère mineur }\end{array}$ & $\begin{array}{l}\text { Exigences pour le diagnostic: } \\
\text { Les } 3 \text { critères majeurs et au moins } 1 \text { critère mineur }\end{array}$ \\
\hline
\end{tabular}

*De nombreux centres canadiens n'analysent pas la masse de globules rouges; par conséquent, le diagnostic repose souvent sur les taux d'hémoglobine, d'hématocrite ou d'érythropoïétine, ainsi que sur les analyses moléculaires.

TE : thrombocytémie essentielle; PV : polycythémie vraie; MFP : myélofibrose primaire; MF : myélofibrose; JAK2 : Janus kinase 2; CALR : calréticuline; LMC : leucémie myéloïde chronique; SMD : syndrome myélodysplasique; LDH : lacticodéshydrogénase

L'ASA est utilisé comme agent oral antiplaquettaire pour réduire le risque de thrombose chez les patients atteints de PV et de TE (Sirhan et al., 2015; Tefferi et Pardanani, 2015, Tefferi et Barbui, 2017; Tonkin et al., 2012; Mesa et al., 2016). Généralement prescrit à faible dosage, il n'est cependant pas indiqué pour le traitement des jeunes patients avec TE à faible risque thrombotique et dont la maladie ne présente pas de mutation du gène JAK2. Les effets secondaires les plus courants de l'ASA sont les saignements, l'indigestion et le risque accru d'ulcère gastroduodénal. Il ne convient pas aux patients sujets aux saignements ou dont le taux de plaquettes est faible ou élevé (Tonkin et al., 2012).

L'hydroxyurée (HU) est un agent oral qui diminue le nombre de cellules sanguines produites par la moelle osseuse en ralentissant la division cellulaire (Tonkin et al., 2012). Généralement recommandé comme traitement cytoréducteur de première ligne dans les cas de PV et de TE, on utilise également ce médicament pour contrôler les symptômes de la splénomégalie et de la thrombocytose chez les patients atteints de MF (Sirhan et al., 2015; Tefferi et Pardanani, 2015, Tefferi et Barbui, 2017; Tonkin et al., 2012; Mesa et al., 2016). L'HU est souvent prescrite en combinaison avec l'ASA aux patients souffrant de TE. Dans les cas de PV, elle est combinée à l'ASA et à la phlébotomie. Ses effets secondaires les plus courants sont, notamment, la cytopénie, ainsi que les ulcères de la jambe et les aphtes buccaux.
Administré par voie sous-cutanée, l'interféron alpha supprime la surproduction de cellules sanguines dans la moelle osseuse. Il est souvent utilisé comme traitement de deuxième ou troisième intention de la TE ou de la PV (Sirhan et al., 2015; Tefferi et Barbui, 2015). Contrairement à l'HU, il n'est pas contre-indiqué pendant la grossesse. Il est donc généralement privilégié pour traiter les jeunes femmes enceintes ou en âge de procréer. Par contre, il provoque souvent d'importants effets secondaires tels que myélosuppression, infection, symptômes grippaux, dépression, diarrhée, vision trouble et asthénie.

Le busulfan est un agent alkylant qui interfère avec la production de cellules sanguines. On utilise souvent le busulfan oral comme traitement de deuxième intention chez les patients atteints d'une NMP sans mutation BCR-ABL1 qui sont intolérants à l'HU ou chez qui l'HU entraîne des effets secondaires (Tefferi et Barbui, 2015). Il est possible que le busulfan cause des nausées, ainsi qu'une cytopénie profonde et prolongée, le plus souvent de nature thrombocytopénique (Begna et al., 2016).

$\mathrm{Au}$ départ employé comme anticoagulant, l'anagrélide (ANA) entraîne une activité intense et spécifique de réduction des plaquettes, dont il diminue la production en inhibant le développement des colonies de mégacaryocytes. C'est un médicament oral utilisé principalement comme traitement de deuxième intention dans les cas de TE pour prévenir la thrombose et l'hémorragie (Birgegård, 2016). Il ne semble pas 


\begin{tabular}{|c|c|c|c|c|}
\hline Traitement & Type de NMP & Indications du traitement & $\begin{array}{l}\text { Événements } \\
\text { indésirables et éléments } \\
\text { de sûreté à prendre en } \\
\text { considération }\end{array}$ & $\begin{array}{l}\text { Suivi/prise en charge des événements indésirables } \\
\text { associés au traitement }\end{array}$ \\
\hline $\begin{array}{l}\text { ASA à faible } \\
\text { dose }(81 \mathrm{mg} / \\
\text { jour) }\end{array}$ & - TE et PV & $\begin{array}{l}\text { - Réduction de l'incidence de } \\
\text { thrombose, particulièrement lorsque } \\
\text { combiné à l'HU }\end{array}$ & $\begin{array}{l}\text { - Risque accru de } \\
\text { saignements } \\
\text { - Risque d'ulcère } \\
\text { gastroduodénal }\end{array}$ & $\begin{array}{l}\text { - Renseignez les patients sur le risque de saignements } \\
\text { et d'ulcère gastroduodénal associé à l'ASA } \\
\text { - Surveillez la numération plaquettaire pendant le } \\
\text { traitement: } \\
\text { - Si }<50000 / \mathrm{m}^{3} \text {, envisager de cesser la prise d'ASA }\end{array}$ \\
\hline $\begin{array}{l}\text { Hydroxyurée } \\
\text { (HU) }\end{array}$ & \begin{tabular}{|l|} 
- TE, PV et \\
MF (s'il y a \\
splénomégalie et \\
thrombocytose \\
symptomatiques)
\end{tabular} & $\begin{array}{l}\text { - Réduction du nombre de cellules } \\
\text { sanguines produites par la moelle } \\
\text { osseuse en ralentissant la division } \\
\text { cellulaire } \\
\text { - Efficace pour prévenir la thrombose } \\
\text { - Généralement utilisé comme } \\
\text { traitement cytoréducteur de première } \\
\text { intention pour la PV et la TE }\end{array}$ & $\begin{array}{l}\text { - Ulcères de la jambe et } \\
\text { aphtes buccaux } \\
\text { - Lésions cutanées } \\
\text { - Anémie } \\
\text { - Neutropénie } \\
\text { - Fièvre }\end{array}$ & $\begin{array}{l}\text { - Surveillez les signes d'ulcères de la jambe ou } \\
\text { d'aphtes buccaux et, si vous en constatez la } \\
\text { présence, consultez le médecin pour savoir s'il } \\
\text { pourrait être nécessaire de réduire la dose ou } \\
\text { d'interrompre le traitement }\end{array}$ \\
\hline Anagrélide & $\cdot \mathrm{TE}$ & $\begin{array}{l}\text { - Activité puissante et spécifique de } \\
\text { diminution des plaquettes } \\
\text { - Réduction de la production } \\
\text { de plaquettes en inhibant le } \\
\text { développement des colonies de } \\
\text { mégacaryocytes }\end{array}$ & $\begin{array}{l}\text { - Céphalée } \\
\text { - Tachycardie } \\
\text { - Augmentation possible } \\
\text { du risque d'hémorragie } \\
\text { lorsque combiné à } \\
\text { l'ASA }\end{array}$ & $\begin{array}{l}\text { - Envisagez des examens cardiaques (fréquence } \\
\text { cardiaque, pouls, ECG) avant et pendant le } \\
\text { traitement si le patient présente un risque élevé } \\
\text { d'événements cardiovasculaires }\end{array}$ \\
\hline Phlébotomie & - PV & $\begin{array}{l}\text { - Une saignée pratiquée pendant la } \\
\text { procédure constitue une méthode } \\
\text { simple pour diminuer l'excès de GR } \\
\text { - Cause une carence en fer chez les } \\
\text { patients en limitant la production } \\
\text { de GR }\end{array}$ & $\begin{array}{l}\text { Minimes, mais peuvent } \\
\text { comprendre : } \\
\text { - ecchymoses au site de } \\
\text { l'intervention } \\
\text { - fatigue } \\
\text { - sentiment de faiblesse }\end{array}$ & $\begin{array}{l}\text { - Assurez-vous que les patients n'ont pas jeûné } \\
\text { avant la procédure } \\
\text { - Assurez-vous que les patients demeurent bien } \\
\text { hydratés après l'opération (il peut être nécessaire } \\
\text { d'administrer un soluté salé) }\end{array}$ \\
\hline Ruxolitinib & - $P V$ et $M F$ & $\begin{array}{l}\text { - Interfère avec les voies des } \\
\text { protéines JAK et STAT qui règlent } \\
\text { la production de cellules sanguines } \\
\text { et jouent un rôle majeur dans le } \\
\text { mécanisme sous-jacent de la PV et } \\
\text { de la MF } \\
\text { - Traitement approuvé pour : } \\
\text { - la PV lorsque le patient ne répond } \\
\text { pas bien à l'HU ou y est intolérant } \\
\text { - traitement de la splénomégalie ou } \\
\text { de ses symptômes associés dans les } \\
\text { cas de MF }\end{array}$ & $\begin{array}{l}\text { - Anémie } \\
\text { - Thrombocytopénie } \\
\text { - Diarrhée } \\
\text { - Spasmes musculaires } \\
\text { - Étourdissements } \\
\text { - Dyspnée }\end{array}$ & $\begin{array}{l}\text { - Pour les patients de plus de } 60 \text { ans, envisagez } \\
\text { le recours au vaccin contre le zona avant le } \\
\text { traitement } \\
\text { - Si le patient présente un risque élevé } \\
\text { d'événements cardiovasculaires, envisagez des } \\
\text { examens cardiaques (fréquence cardiaque, pouls, } \\
\text { ECG) avant et pendant le traitement } \\
\text { - Envisagez un dépistage de la tuberculose avant le } \\
\text { traitement chez les patients à risque élevé } \\
\text { - Surveillez le taux de Hb et la numération } \\
\text { plaquettaire: } \\
\text { - Si la numération plaquettaire est }<125000 / \\
\text { mm , mais }>50000 / \mathrm{mm}^{3}, \text { il pourrait être } \\
\text { nécessaire de réduire la dose. } \\
\text { - Si la numération plaquettaire est }<50000 / \mathrm{mm}^{3} \text {, } \\
\text { il est recommandé d'interrompre le traitement } \\
\text { jusqu'à ce qu'elle revienne à la normale. }\end{array}$ \\
\hline
\end{tabular}

TE : thrombocytémie essentielle; PV : polycythémie vraie; MF : myélofibrose; CV : cardiovasculaire; $H U$ : hydroxyurée; Hb : hémoglobine; ECG : électrocardiogramme; GR : globules rouges; ASA : acide acétylsalycylique; TB : tuberculose. 
empêcher la fibrose et, vu ses propriétés anticoagulantes, il peut augmenter le risque d'hémorragie lorsqu'il est combiné à l'ASA. Ses effets secondaires les plus courants sont les céphalées et la tachycardie, mais ces effets se résorbent souvent après les premières semaines de traitement.

Administré par voie orale, le ruxolitinib est un inhibiteur des protéines Janus kinase 1 et 2 (JAK1 et JAK2). Il s'agit d'un traitement ciblé qui interfère avec les protéines JAK et le signal transducteur et activateur de la transcription (protéines STAT) qui règle la production de cellules sanguines et dont on connaît le rôle fondamental dans les mécanismes sous-jacents de la PV et de la MF. Le ruxolitinib s'est révélé efficace pour réduire le volume de la rate, soulager les symptômes constitutionnels, améliorer la qualité de vie et stabiliser la fibrose. Il peut également améliorer la longévité (Verstovsek et al., 2012, 2016; Harrison et al., 2012; Harrison et al., 2016; Vannucchi et al., 2015; Passamonti et al., 2015). Au Canada, le ruxolitinib est approuvé pour le traitement des patients souffrant de PV qui réagissent mal à l'HU ou qui y sont intolérants, de même que pour le traitement de la splénomégalie et des symptômes associés chez les adultes atteints de MF primaire, de MF post-PV ou de MF post-TE (monographie du ruxolitinib, 2017). Parmi les effets secondaires les plus courants du traitement au ruxolitinib, on retrouve l'anémie dose-dépendante et la thrombocytopénie.

Bien qu'ils ne figurent pas en détail dans le présent article, d'autres traitements pharmacologiques sont utilisés pour traiter la MF, par exemple l'érythropoïétine, les corticostéroïdes et les immunomodulateurs pour le traitement de l'anémie.

\section{RÔLE DE L'INFIRMIÈRE DANS LE TRAITEMENT DES NMP}

Pour assurer la conformité au traitement et en optimiser les résultats, les infirmières jouent un rôle important : elles établissent les objectifs thérapeutiques, enseignent aux patients comment s'administrer leur traitement et leur expliquent les effets secondaires possibles, en plus de surveiller et de gérer les événements indésirables associés aux traitements des NMP.

Pour les patients sous HU, les infirmières doivent surveiller les signes d'ulcères de la jambe ou d'aphtes buccaux et consulter le médecin pour réduire la dose ou interrompre le traitement si elles en constatent l'apparition. Aux patients sous interféron, elles doivent enseigner la bonne technique d'injection sous-cutanée, de même que des stratégies pour réduire les symptômes grippaux, notamment en buvant davantage, en prenant des analgésiques et des antipyrétiques et en gardant le lit jusqu'à ce que les symptômes se résorbent. Dans les cas de phlébotomie, elles doivent s'assurer que le patient n'a pas jeûné avant la procédure et qu'il reste bien hydraté après l'opération.

Il est également important pour les infirmières de surveiller le taux de plaquettes des patients qui prennent de l'ASA, du busulfan ou du ruxolitinib pour déterminer s'il convient de réduire la dose ou d'interrompre le traitement. Les effets hématologiques du ruxolitinib peuvent généralement être traités par la modification de la dose et l'interruption temporaire $\mathrm{du}$ traitement, ainsi que par transfusions de globules rouges en cas d'anémie (Mesa et Cortes, 2013).

Le tableau 5 présente un résumé des traitements couramment utilisés pour traiter les NMP, de même que les effets secondaires associés et les stratégies infirmières de suivi et de traitement de ces événements indésirables.

\section{CONCLUSION}

De nos jours, les patients atteints de NMP sont bien informés grâce aux nombreuses ressources disponibles en ligne. Les infirmières doivent donc bien comprendre les caractéristiques, le diagnostic et le traitement de ces maladies. À titre de personne-ressource principale, l'infirmière joue un rôle essentiel pour communiquer avec les patients souffrant de NMP, pour les renseigner sur les possibilités de traitement, et pour surveiller et gérer les événements indésirables des traitements afin d'en maximiser l'observance et les résultats. Idéalement, tous les patients atteints de NMP devraient être pris en charge selon un modèle de soins partagés, avec une étroite collaboration entre les infirmières, un hémato-oncologue en milieu communautaire et un centre de soins tertiaires possédant une expertise dans le domaine des NMP.

\section{CONFLIT D'INTÉRÊTS}

Novartis a versé des honoraires à : Sabrina Fowlkes, pour des conférences, l'élaboration d'un programme éducatif et son rôle d'infirmière-consultante; à Cindy Murray, pour des activités éducatives; Adrienne Fulford, pour une conférence et une rencontre de consultation; Tammy De Gelder, pour des conférences, des activités éducatives et de la consultation; Nancy Siddiq, pour des activités éducatives. Les auteures n'ont pas été rémunérées pour la rédaction du présent article. 


\section{RÉFÉRENCES}

Abdel-Wahab, O.I., \& Levine, R.L. (2009). Primary myelofibrosis: Update on definition, pathogenesis, and treatment. Annual Review of Medicine, 60, 233-45.

Arber, D.A., Orazi, A., Hasserjian, R., Thiele, J., Borowitz, M.J., Le Beau, M.M., Bloomfield, ... Vardiman, J.W. (2016). The 2016 revision to the World Health Organization classification of myeloid neoplasms and acute leukemia. Blood, 127(20), 2391-2405.

Begna, K., Abdelatif, A., Schwager, S., Hanson, C., Pardanani, A., \& Tefferi A. (2016). Busulfan for the treatment of myeloproliferative neoplasms: The Mayo Clinic experience. Blood Cancer Journal, 6, e427.

Birgegård, G. (2016). The use of anagrelide in myeloproliferative neoplasms, with focus on essential thrombocythemia. Current Hematologic Malignancy Reports, 11(5), 348-355.

Cervantes, F., Dupriez, B., Pereira, A., Passamonti, F., Reilly, J.T., Morra, E., Vannucchi, A.M., ... Tefferi, A. (2009). New prognostic scoring system for primary myelofibrosis based on a study of the International Working Group for Myelofibrosis Research and Treatment. Blood, 113(13), 2895-2901.

Finazzi, G., \& Barbui, T. (2007). How I treat patients with polycythemia vera. Blood, 109(12), 5104-11.

Gangat, N., Caramazza, D., Vaidya, R., George, G., Begna, K., Schwager, S., Van Dyke, D., ... Tefferi, A. (2011). DIPSS plus: A refined Dynamic International Prognostic Scoring System for primary myelofibrosis that incorporates prognostic information from karyotype, platelet count, and transfusion status. Journal of Clinical Oncology, 29(4), 392-397.

Geyer, H.L., \& Mesa, R.A. (2014). Therapy for myeloproliferative neoplasms: When, which agent, and how? Blood, 124(24), 3529-37.

Harrison, C., Kiladjian, J.J., Al-Ali, H.K., Gisslinger, H., Waltzman, R., Stalbovskaya, V., McQuitty, M., ... Barosi, G. (2012). JAK inhibition with ruxolitinib versus best available therapy for myelofibrosis. New England Journal of Medicine, 366(9), 787-98.

Harrison, C.N., Vannucchi, A.M., Kiladjian, J.J., Al-Ali, H.K., Gisslinger, H., Knoops, L., Cervantes, F., ... Barbui, T. (2016). Longterm findings from COMFORT-II, a phase 3 study of ruxolitinib versus best available therapy for myelofibrosis. Leukemia, 30, 1701-1707.

Mesa, R.A., \& Cortes, J. (2013). Optimizing the management of ruxolitinib in patients with myelofibrosis: the need for individualized dosing. Journal of Hematology a Oncology, 6, 79.

Mesa, R., Jamieson, C., Bhatia, R., Deininger, M.W., Gerds, A.T., Gojo, I., Gotlib, J., ... Sundar, H. (2016). Myeloproliferative Neoplasms, Version 2.2017, NCCN Clinical Practice Guidelines in Oncology. Journal of the National Comprehensive Cancer Network, 14(12), 1572-1611.

Nangalia, J., \& Green, T.R. (2014) The evolving genomic landscape of myeloproliferative neoplasms. Hematology. American Society of Hematology. Education Program, 2014, 287-296.

Passamonti, F., Cervantes, F, Vannucchi, A.M., Morra, E., Rumi, E., Pereira, A., Guglielmelli, P., ... Tefferi, A. (2010). A dynamic prognostic model to predict survival in primary myelofibrosis: a study by the IWG-MRT (International Working Group for Myeloproliferative Neoplasms Research and Treatment). Blood, 115(9), 1703-1708.
Passamonti, F., Maffioli, M., Caramazza, D., \& Cazzola M. (2011) Myeloproliferative neoplasms: from JAK2 mutations discovery to JAK2 inhibitor therapies. Oncotarget, 2(6), 485-490.

Passamonti, F., Vannucchi, A.M., Cervantes, F., Harrison, C., Morra, E., Kantarjian, H., \& Verstovsek, S. (2015). Ruxolitinib and survival improvement in patients with myelofibrosis. Leukemia, 29(3), 739-40.

Radaelli, F., Onida, F., Rossi, F.G., Zilioli, V.R., Colombi, M., Usardi, P., Calori, R., \& Zanella, A. (2008). Second malignancies in essential thrombocythemia (ET): A retrospective analysis of 331 patients with long-term follow-up from a single institution. Hematology, 13(4), 195-202.

Rollison, D.E., Howlader, N., Smith, M.T., Strom, S.S., Merritt, W.D., Ries, L.A., Edwards, B.K., \& List, A.F. (2008). Epidemiology of myelodysplastic syndromes and chronic myeloproliferative disorders in the United States, 2001-2004, using data from the NAACCR and SEER programs. Blood, 112(1), 45-52.

Ruxolitinib (Jakavi) Product Monograph. Novartis Pharmaceuticals Canada, Inc. March 2, 2017.

Sirhan, S., Busque, L., Foltz, L., Grewal, K., Hamm, C., Laferriere, N., Laneuville, P., ... Gupta, V. (2015). Evolving therapeutic options for polycythemia vera: Perspectives of the Canadian Myeloproliferative Neoplasms Group. Clinical Lymphoma, Myeloma Q Leukemia, 15, 715-27.

Tefferi, A., \& Barbui, T. (2015). Polycythemia vera and essential thrombocythemia: 2015 update on diagnosis, risk-stratification and management. American Journal of Hematology, 90(2), 162-73.

Tefferi, A., \& Barbui, T. (2017). Polycythemia vera and essential thrombocythemia: 2017 update on diagnosis, risk-stratification, and management. American Journal of Hematology, 92(1), 94-108.

Tefferi, A., \& Pardanani, A. (2015). Myeloproliferative neoplasms: a contemporary review. JAMA Oncology, 1(1), 97-105.

Tefferi, A., Rumi, E., Finazzi, G., Gisslinger, H., Vannucchi, A.M., Rodeghiero, F., Randi, M.L., ... Barbui, T. (2013). Survival and prognosis among 1545 patients with contemporary polycythemia vera: an international study. Leukemia, 27(9), 1874-1881.

Tonkin, J., Francis, Y., Pattinson, A., Peters, T., Taylor, M., Thompson, R., \& Wallis, L. (2012). Myeloproliferative neoplasms: Diagnosis, management and treatment. Nursing Standard, 26(51), 44-51.

Vannucchi, A.M., Kiladjian, J,J., Griesshammer, M., Masszi, T., Durrant, S., Passamonti, F., Harrison, C.N., ... Verstovsek, S. (2015). Ruxolitinib versus standard therapy for the treatment of polycythemia vera. New England Journal of Medicine, 372(5), 426-435.

Verstovsek, S., Mesa, R.A., Gotlib, J., Levy, R.S., Gupta, V., DiPersio, J.F., Catalano, J.V., ... Kantarjian, H.M. (2012). A double-blind, placebo-controlled trial of ruxolitinib for myelofibrosis. New England Journal of Medicine, 366(9), 799-807.

Verstovsek, S., Vannucchi, A.M., Griesshammer, M., Masszi, T., Durrant, S., Passamonti, F., Harrison, C.N., ... Kiladjian, J.J. (2016). Ruxolitinib versus best available therapy in patients with polycythemia vera: 80-week follow-up from the RESPONSE trial. Haematologica, 101(7), 821-9.

Wolanskyj, A.P., Schwager, S.M., McClure, R.F., Larson, D.R., \& Tefferi, A. (2006). Essential thrombocythemia beyond the first decade: life expectancy, long-term complication rates, and prognostic factors. Mayo Clinic Proceedings, 81(2), 159-66. 